\title{
Structure Guided Drug Designing Coupled to Advanced Molecular Dynamics Revealed Differential Binding Properties (Affinity/Stability) of Celecoxib and Niflumic Acid in the Active Site of Cyclooxygenase-2 Enzyme
}

shabir ahmad ganai ( $\square$ shabir.muntazir82@gmail.com )

University of Kashmir

\section{Basit Amin Shah}

University of Kashmir

Asif Lone

University of Delhi

\section{Faieza Arwa}

SKUAST Jammu: Sher-e-Kashmir University of Agricultural Sciences and Technology of Jammu

Firdose Ahmad Malik

SKUAST Kashmir: Sher-E-Kashmir University of Agricultural Sciences and Technology Kashmir

\section{Research Article}

Keywords: COX-2, Niflumic acid, Celecoxib, Molecular docking, MM-GBSA, Molecular dynamics

Posted Date: February 7th, 2022

DOI: https://doi.org/10.21203/rs.3.rs-1308507/v1

License: (c) (1) This work is licensed under a Creative Commons Attribution 4.0 International License.

Read Full License 


\section{Abstract}

Cyclooxygenase-2 (COX-2) has aggressive implications in inflammation triggered disorders such as neurodegeneration and cancer. Among the various inhibitors available for this enzyme celecoxib and niflumic acid are considered as selective inhibitors. These inhibitors have shown in vitro assays have been observed to hamper the COX-2 activity. However, the structural studies at atomistic depth shedding light on diverse aspects of these inhibitors in the binding groove of COX-2 have not been addressed. Thus, in this study we employed structure-governed drug designing approach in concert with real-time molecular dynamics for unravelling the binding likeliness, interaction mechanism and stability of niflumic acid and celecoxib in the COX-2 active site. Our findings indicate the relatively higher binding proclivity and stability of niflumic acid in the active site of COX-2 as compared to celecoxib. Although these inhibitors bind at the same COX-2 site but they revealed differential interaction profile. The outcome from this study will serve as a point of departure for designing novel COX-2 inhibitors with improved potency, selectivity and stability for their subsequent use to vanquish vicious inflammation backed disorders.

\section{Introduction}

It is quite established that mature human cyclooxygenase-2 contains 587 amino acid residues [1]. This enzyme also known as prostaglandin G/H synthase 2 is encoded by PTGS2 gene [2]. The 17 amino acid residue signal peptide at its amino-terminal end is removed during post-translational processing due to which its final amino acid residue count decreases from 604 to 587 [1]. Cyclooxygenase enzymes catalyse the critical step in generating prostaglandin $\mathrm{H} 2$ ( $\mathrm{PGH} 2)$ from arachidonic acid. Once PGH2 is synthesized it serves as a precursor for leukotrienes, prostacyclins and thromboxanes [3]. Prostaglandins play multiple roles as they act as immune system regulators, maintain the integrity of gastrointestinal system apart from kidney development [4]. Among the cyclooxygenases COX-1 and COX-2 isozymes are worth interesting as they have strong cross-talk with various physiological and pathological processes [5]. While COX-1 manifests constitutive expression, COX-2 is inducible as it exhibits prompt expression in a variety of cell types in reciprocation to pro-inflammatory molecules, growth factors and cytokines [6]. Due to its central role in inciting inflammation in peripheral tissues this enzyme has emerged as a potential therapeutic target to bridle inflammation [7]. Mounting evidences suggest that COX-2 has severe implications in cancer, multidrug resistance and neurodegenerative diseases [8-10]. Thus inhibitors of COX-2 which were conventionally considered as anti-inflammatory molecules are now gaining importance in anticancer and anti-neurodegenerative therapy. Traditional drugs such as ibuprofen, naproxen and aspirin used for pharmacological intervention of this enzyme due to lack of selectivity also target COX-1, the cytoprotective isozyme culminating in gastrointestinal and ulcerogenic side effects [5, 11]. Celecoxib, a selective non-steroidal COX-2 inhibitor has shown promising results in inflammationinduced diseases. This selective inhibitor was found to provoke apoptosis in prostate carcinoma cells through obstruction of Akt activation [12]. In human cervical cancer cells celecoxib induced apoptosis and cell cycle arrest [13]. Further, in various breast cancer cells and in hepatocellular carcinoma cells (positive for hepatitis $B$ virus $X$ protein) celecoxib provoked apoptotic type cell cytotoxicity $[14,15]$. 
Niflumic acid, another COX-2 inhibitor has been reported to alleviate the production of kynurenic acid in the cortex of rat brain under conditions of in vitro [16]. Niflumic acid like celecoxib is selective COX-2 inhibitor. While niflumic acid manifested an $\mathrm{EC}_{50}$ value of $5.4 \mu \mathrm{M}$ against $\mathrm{COX}-2$, the defined value for COX-1 was found to be $25 \mu \mathrm{M}$ [17]. Despite these findings no structural studies at atomistic level have been performed for exploring the binding propensity, interaction mechanism and stability of celecoxib and niflumic acid in the active site of COX-2 enzyme. Thus, we focussed on delineating the binding inclination and stability of these selective inhibitors against therapeutically relevant COX-2 using molecular docking in extra-exactitude flexible mode, molecular mechanics generalized born surface area (MM-GBSA) approach. Importantly, the stability of celecoxib and niflumic acid in the binding pocket of COX-2 was monitored in real time fashion using the advanced molecular dynamics approach.

\section{Methods And Techniques Used}

\section{Cognate docking}

For testing the docking algorithm for reproducing the ligand pose solved through $\mathrm{x}$-ray crystallography cognate or self-docking protocol was followed. Crystal structure coordinates of Cyclooxygenase-2 (COX2) bearing a four letter code 5IKT were fetched from widely used Protein Data Bank (PDB) [18]. The structure was prepared through the popular Protein Preparation Wizard of globally famous Schrödinger package. As a thumb rule the missing residues and side chains were recovered. Water molecules present in the structure were removed or kept as per the certified guidelines. Coordinates of heteroatoms except the native ligand were deleted from the COX-2 coordinate file. The structure was then optimized and this was followed by minimization of the structure. At the end of minimization the PDB file of COX-2 was duplicated. Among these one of the file was used for specification of active site for which the help of native ligand was taken. The native ligand was then docked against the grid-demarcated COX-2 using the Glide docking procedure in high-precison mode. Following docking, the root mean square deviation (RMSD) was estimated between the algorithm docked pose and the pose that has been determined through experimental method.

\section{Molecular docking and interaction profile}

As aforesaid, coordinates of COX-2 were retrieved from Protein Data Bank (PDB ID: 5IKT) [18]. The coordinates of celecoxib and niflumic acid were fetched from the large small molecule database known as PubChem. While celecoxib possessed a PubChem CID: 2662 niflumic acid portrayed the defined ID as 4488 [19]. The protein preparation has already mentioned in cognate docking section. The ligand molecules were made suitable for docking against COX-2 with the help of another widely accepted LigPrep tool $[20,21]$. Identical conditions were maintained during the preparation of niflumic acid and celecoxib. These ligands were minimized, desalted and chiralities were treated according to certified protocol. All the prepared ligands were flexible docked against COX-2 using the extra-precison mode of pandemically accepted Glide tool. As docking score integrates epik penalties thus this score was preferred instead of another score termed as Glide Score [22-24]. A recent tool protein ligand interaction 
profiler (PLIP) was used for finding interactions between celecoxib-COX-2 and niflumic acid-COX-2. This tool is highly extensive and has the ability to detect a broader range of interactions in ligand-protein docked complexes. PLIP simply requires the best pose of ligand in association with the receptor protein for providing the interaction details $[22,25]$.

\section{Binding Free energy measurement}

The two ligand-COX-2 docked complexes were subjected to various energy calculations by employing the molecular mechanics generalized born surface area (MMGBSA) technique [22]. This method has performed comparatively better in measuring the relative binding affinities of ligands as evidenced from various studies. Frozen receptor conditions were maintained during calculations as these conditions have shown better correlation with experimental findings [26]. MMGBSA method takes the advantage of VSGB solvation model and performs the variety of energy calculations [27]. From these calculations the binding energy was calculated using the equation discussed previously. Least negative value indicates least binding affinity whereas highest binding affinity is represented by more negative values [28]. The following equating was used for calculating the relative binding free energy values;

\section{$E_{\text {Complex (minimized) }}-E_{\text {Ligand (minimized) }}-E_{\text {Receptor (minimized) }}=\Delta G_{(\text {bind })}$}

\section{Futuristic Classical Molecular Dynamics Simulation}

Celecoxib-COX2 and niflumic acid-COX2 complexes were subjected to molecular dynamics simulation for 10 ns using the world famous Desmond software [29]. Molecular dynamics sheds light on stability of ligands in the binding pocket of enzymes and has significant advantages over the static molecular docking approaches. First the setup files were produced through the Desmond's system builder option. Solvation of Celecoxib-COX2 and niflumic acid-COX2 complexes was done by employing the water model namely TIP4P [30, 31]. This was followed by setting boundary conditions and subsequent minimizing of box volume. Both the systems were neutralized by adding correct number of counter ions. Besides, the salt was added to both systems as per standard protocol. The core molecular dynamics was performed using the molecular dynamics aspect of Desmond. Constant pressure, temperature and number of atoms were kept all through simulation. While pressure was kept 1.0 bar, temperature was maintained at $300 \mathrm{~K}$ using the proper algorithms [32,33]. However, before initiating molecular dynamics the systems were minimized as per standard guidelines. The information enclosed in the simulation output file was extracted using the simulation interaction diagram (SID). While global fluctuations were measured by RMSD of protein, the local fluctuations were indicated by RMSF values. Ligand RMSD, the indicator of ligand stability in docked state with COX2 was also calculated throughout the simulation time. Further, the interactions fraction and percentage time for which an interaction was maintained between receptor and ligand atoms was estimated [28].

\section{Results And Discussion}


Before the onset of real molecular docking we tested the algorithm for its ability to detect the active site properly and to reproduce the experimentally solved pose of the ligand molecule. After redocking the cocrystallized or native ligand of the COX-2 enzyme, we carefully quantified the RMSD between the two ligand poses (one being experimentally derived pose and another being algorithm generated). The algorithm proved to detect active site very accurately and was quite capable of emulating the experimental (crystal) pose of the ligand [28]. This conclusion has been derived as the RMSD value obtained was $0.4048 \AA$ (Figure 1). RMSD values ranging from 0 to $2 \AA$ are quite acceptable in small molecule docking studies [28,34]. After assuring the precision and accuracy of docking algorithm we moved to actual molecular docking for ranking the ligand molecules.

\section{Niflumic acid showed comparably more docking score against COX-2}

Among the docked molecules, niflumic acid showed more negative value of docking score against COX-2 as compared to celecoxib. More negative docking score of niflumic acid indicates its stronger inclination towards this enzyme [34]. While celecoxib showed a docking score of -9.31 , niflumic acid displayed this score as -10 indicating its relatively stronger binding propensity (Figure 2 ). As a regular procedure it is common to supplement the findings of one experiment with other for extra surety thus in the subsequent experiment we tried to explore the binding free energy of aforementioned inhibitors with more reliable technique.

\section{MMGBSA study further supported the molecular docking accuracy}

Two MM-GBSA experiments were performed for estimating the binding free energy values of docked complexes. Niflumic acid like docking score showed more negative value (lowest score) of binding free energy than celecoxib. While a binding free energy value of $-39.5573 \mathrm{kcal} / \mathrm{mol}$ was manifested by celecoxib-COX-2 complex, a more negative value was demonstrated by niflumic acid-COX-2 complex $(-54.9838 \mathrm{kcal} / \mathrm{mol})$. More negative values of binding free energy in case of niflumic acid-COX-2 indicate stronger interactions than celecoxib-COX-2 (Figure 3) [34]. Now it is evident that niflumic acid showed not only more negative value of docking score but also more binding free energy value against COX-2 in comparison to celecoxib. Till here we proved through molecular docking and MMGBSA method that niflumic acid has relatively favourable affinity over celecoxib against COX-2. In the forthcoming experiments we are going to scrutinize the interaction of these inhibitors individually in docked state with cOX-2.

\section{Celecoxib showed more interactions over niflumic acid}

Various types of interactions are known to exist between protein receptors and drug molecules. Among these interactions hydrophobic contacts, hydrogen bonds, pi-pi, pi-cation and salt bridges are prominent. Niflumic acid showed seven hydrophobic and two hydrogen bonding interactions with active site residues of COX-2. Among the residues showing hydrophobic contacts LEU 352, TYR 355, TYR 385, TRP 387, VAL 523 and ALA 527 were prominent. Among the hydrogen bonding displaying residues TYR 385 and SER 530 were noticeable. On the other hand celecoxib demonstrated ten hydrophobic and five hydrogen 
bonds with COX2. Apart from this, one halogen bond was seen with ARG 120 of this enzyme. PHE 518 showed dual contacts (hydrophobic and hydrogen bond) with celecoxib (Figure 4). Thus niflumic acid and celecoxib showed similar but surely not identical interaction profile in the bound state with this inflammation facilitating enzyme. The differences in interaction profile can be ascribed to structural differences between these inhibitors giving rise to distinct interactions within the COX-2 pocket [30].

After sequential binding proclivity and interaction studies we focussed on dynamic study which can answer the stability of niflumic acid/celecoxib in the binding pocket of COX-2. Thus, in the upcoming steps we employed molecular dynamics simulation approach to address this question.

\section{Niflumic acid showed comparatively more stability in the COX-2 active site}

It is well known that binding of ligands to proteins is dynamic thereby involving various dynamic and complex transitions. While a flexible ligand can alter its conformation on binding the target site in a best possible manner, the protein can change its dynamics and conformation for promoting ligand binding [35]. Thus it is obvious that static approaches like molecular docking should be coupled to dynamic approaches like molecular dynamics simulations for gaining more insights about ligand-receptor stability $[28,32]$. We subjected celecoxib-COX-2 complex and niflumic acid-COX-2 for 10 ns simulation using the Desmond software. Even molecular dynamics simulation supplemented the molecular docking and MMGBSA conclusions. Niflumic acid demonstrated relatively better stability in the binding pocket of COX2 as compared to celecoxib. This crux has been taken as niflumic acid showed comparatively lesser fluctuations indicated by the lower RMSD values than celecoxib (Figure 5) [36]. Further, the COX-2 showed lesser local fluctuations in docked state with niflumic acid than with celecoxib as indicated by the RMSF values (Figure 6). Residues of COX-2 crucial for interaction with celecoxib and niflumic acid were also explored by analysing the respective trajectory. Certain residues including SER 530 and TYR 385 were critical in interacting with niflumic acid whereas residues like LEU 352 and ARG 120 were crucial in case of celecoxib (Figure 7). Importantly, one interactions of niflumic acid sustained for $99 \%$ (TYR 385) of the simulation time while in case of celecoxib-COX-2 only one interaction remained for 91\% (LEU 352) of the simulation duration which again favours stability of niflumic acid over celecoxib (Figure 8).

\section{Conclusion}

This work delineated the binding tendency, interaction parameters and stability of two selective nonsteroidal inhibitors against COX2. Although their ability of inhibiting COX-2 in cell based and other assays was well established, their comparative and atomistic scale structure based study with COX-2 was entirely unaddressed. Thus, we coupled structure based drug designing tactics with futuristic molecular dynamics approach to answer many unsolved questions. In this study we proved that niflumic acid in comparison to celecoxib shows more binding inclination towards COX-2. This crux is supported by lowest values of docking and binding free energy values of niflumic acid over celecoxib. Though the two inhibitors bind at the same site in COX-2 they showed similar but obviously non-identical interaction mechanism with this enzyme. The above statement is supported by more number of hydrogen, 
hydrophobic and presence of halogen bond in case of celecoxib. Most importantly, niflumic acid evinced more stability in the binding site of COX-2 as evident from the RMSD, RMSF and the persistence of interactions throughout the simulation. The output from this study provides the critical insights which will be highly helpful for designing novel COX-2 inhibitors with improved potency and stability for effective treatment of COX-2 triggered human disorders.

\section{Declarations}

Funding: Science and Engineering Research Board.

Conflicts of interest/Competing interests: All the authors endorse the submission and have no conflict of interest.

Availability of data and material: All the data has been submitted as Figures with the manuscript.

Code availability: NA.

Authors' contributions: Ganai SA designed the work. Ganai SA, Shah BA did main work. Lone A, Arwa F and Malik FA substantially assisted in the work. Ganai SA wrote the manuscript.

\section{References}

1. Knights KM et al (2010) Defining the COX inhibitor selectivity of NSAIDs: implications for understanding toxicity. Expert Rev Clin Pharmacol 3(6):769-776

2. Ulrich CM et al (2005) PTGS2 (COX-2) -765G > C promoter variant reduces risk of colorectal adenoma among nonusers of nonsteroidal anti-inflammatory drugs. Cancer Epidemiol Biomarkers Prev 14(3):616-619

3. Granström E (1984) The arachidonic acid cascade. The prostaglandins, thromboxanes and leukotrienes. Inflammation 8 Suppl, S15-25

4. Williams CS et al (1999) The role of cyclooxygenases in inflammation, cancer, and development. Oncogene 18(55):7908-7916

5. Bhardwaj A et al (2017) In situ click chemistry generation of cyclooxygenase-2 inhibitors. Nat Commun 8(1):1

6. Zidar $\mathrm{N}$ et al (2009) Cyclooxygenase in normal human tissues-is COX-1 really a constitutive isoform, and COX-2 an inducible isoform? J Cell Mol Med 13(9B):3753-3763

7. Minghetti L (2004) Cyclooxygenase-2 (COX-2) in inflammatory and degenerative brain diseases. $J$ Neuropathol Exp Neurol 63(9):901-910

8. Sui H et al (2011) COX-2 contributes to P-glycoprotein-mediated multidrug resistance via phosphorylation of c-Jun at Ser63/73 in colorectal cancer. Carcinogenesis 32(5):667-675 
9. Pang LY et al (2016) Cyclooxygenase-2: A Role in Cancer Stem Cell Survival and Repopulation of Cancer Cells during Therapy. Stem cells international 2016, 2048731-2048731

10. Minghetti $L$ (2007) Role of COX-2 in inflammatory and degenerative brain diseases. Subcell Biochem 42:127-141

11. Zhu X-T et al (2018) Selective COX-2 inhibitor versus non-selective COX-2 inhibitor for the prevention of heterotopic ossification after total hip arthroplasty: A meta-analysis. Medicine 97:31

12. Hsu AL et al (2000) The cyclooxygenase-2 inhibitor celecoxib induces apoptosis by blocking Akt activation in human prostate cancer cells independently of Bcl-2. J Biol Chem 275(15):11397-11403

13. Setiawati A, Setiawati A (2016) Celecoxib, a COX-2 Selective Inhibitor, Induces Cell Cycle Arrest at the G2/M Phase in HeLa Cervical Cancer Cells. Asian Pac J Cancer Prev 17(4):1655-1660

14. Xie H et al (2009) Potent cell growth inhibitory effects in hepatitis B virus X protein positive hepatocellular carcinoma cells by the selective cyclooxygenase- 2 inhibitor celecoxib. Mol Carcinog 48(1):56-65

15. Wang $\mathrm{G}$ et al (2017) Celecoxib induced apoptosis against different breast cancer cell lines by downregulated NF-KB pathway. Biochem Biophys Res Commun 490(3):969-976

16. Zakrocka I et al (2019) Influence of Cyclooxygenase-2 Inhibitors on Kynurenic Acid Production in Rat Brain in Vitro. Neurotox Res 35(1):244-254

17. Čeponytė $U$ et al (2018) Comparison of NSAIDs activity in COX-2 expressing and non-expressing 2D and 3D pancreatic cancer cell cultures. Cancer Manag Res 10:1543-1551

18. Orlando BJ, Malkowski MG (2016) Substrate-selective Inhibition of Cyclooxygeanse-2 by Fenamic Acid Derivatives Is Dependent on Peroxide Tone. J Biol Chem 291(29):15069-15081

19. Kim S et al (2020) PubChem in 2021: new data content and improved web interfaces. Nucleic Acids Res 49(D1):D1388-D1395

20. LigPrep S, (Schrödinger LLC, New York LLC (2021) Schrödinger Release 2021-1: LigPrep, Schrödinger, LLC, New York, NY, 2021

21. Madhavi Sastry G et al (2013) Protein and ligand preparation: parameters, protocols, and influence on virtual screening enrichments. J Comput Aided Mol Des 27(3):221-234

22. Ganai SA (2021) Characterizing binding intensity and energetic features of histone deacetylase inhibitor pracinostat towards class I HDAC isozymes through futuristic drug designing strategy. In Silico Pharmacol 9(1):18

23. Friesner RA et al (2006) Extra Precision Glide: Docking and Scoring Incorporating a Model of Hydrophobic Enclosure for Protein-Ligand Complexes. J Med Chem 49(21):6177-6196

24. Ganai SA et al (2021) Delineating binding potential, stability of Sulforaphane-N-acetyl-cysteine in the active site of histone deacetylase 2 and testing its cytotoxicity against distinct cancer lines through stringent molecular dynamics, DFT and cell-based assays. Chem Biol Drug Des 98(3):363-376

25. Salentin S et al (2015) PLIP: fully automated protein-ligand interaction profiler. Nucleic Acids Res 43(W1):W443-W447 
26. Vilar S et al (2010) Ligand and structure-based models for the prediction of ligand-receptor affinities and virtual screenings: Development and application to the beta(2)-adrenergic receptor. J Comput Chem 31(4):707-720

27. Li J et al (2011) The VSGB 2.0 model: a next generation energy model for high resolution protein structure modeling. Proteins 79(10):2794-2812

28. Ganai SA et al (2017) Combinatorial In Silico Strategy towards Identifying Potential Hotspots during Inhibition of Structurally Identical HDAC1 and HDAC2 Enzymes for Effective Chemotherapy against Neurological Disorders. Front Mol Neurosci 10:357

29. Bowers KJ et al (2006) Scalable Algorithms for Molecular Dynamics Simulations on Commodity Clusters, SC '06: Proceedings of the 2006 ACM/IEEE Conference on Supercomputing, pp. 43-43

30. Ganai SA et al (2018) In silico approaches for investigating the binding propensity of apigenin and luteolin against class I HDAC isoforms. Future Med Chem 10(16):1925-1945

31. Jorgensen WL et al (1983) Comparison of simple potential functions for simulating liquid water. J Chem Phys 79(2):926-935

32. Rai $\mathrm{H}$ et al (2021) Molecular docking, binding mode analysis, molecular dynamics, and prediction of ADMET/toxicity properties of selective potential antiviral agents against SARS-CoV-2 main protease: an effort toward drug repurposing to combat COVID-19. Molecular Diversity.

33. Shah BA et al (2021) Exploring novel and potent molecules for disrupting DEPTOR-mTOR interaction through structure-steered screening, extra-exactitude molecular docking, prime binding free energy estimation and voguish molecular dynamics.J Biomol Struct Dyn,1-11

34. Mir MA et al (2020) Isolation, purification and characterization of naturally derived Crocetin beta-dglucosyl ester from Crocus sativus L. against breast cancer and its binding chemistry with ERalpha/HDAC2. Saudi J Biol Sci 27(3):975-984

35. Hughes TS et al (2012) Ligand and receptor dynamics contribute to the mechanism of graded PPARY agonism. Structure (London, England: 1993) 20 (1), 139-150

36. Das $P$ et al (2020) In-Silico approach for identification of effective and stable inhibitors for COVID-19 main protease ( $M($ pro) ) from flavonoid based phytochemical constituents of Calendula officinalis.Journal of biomolecular structure \& dynamics,1-16

\section{Figures}




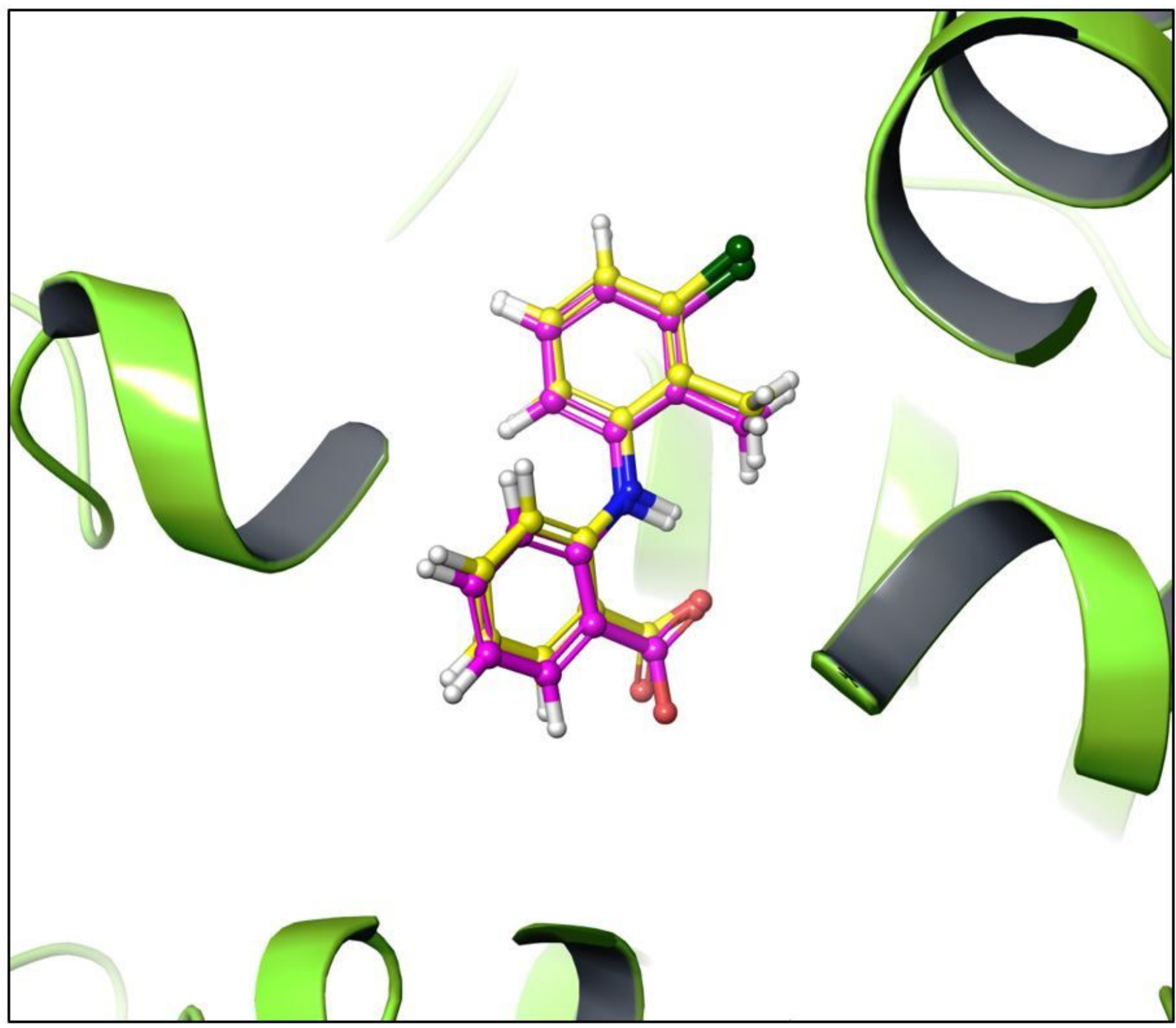

Figure 1

Experimental and redocked pose of tolfenamic acid (native ligand) in the active site of COX-2. The native pose has been shown in pink while the yellow colour indicates redocked pose. RMSD between the two poses was estimated to be $0.4048 \AA$ certifying the docking algorithms ability to generate experimentally proved pose quite precisely. RMSD values ranging from 0 to $2 \AA$ are permissible. This entire information is the outcome of cognate docking which is also known as self-docking. 


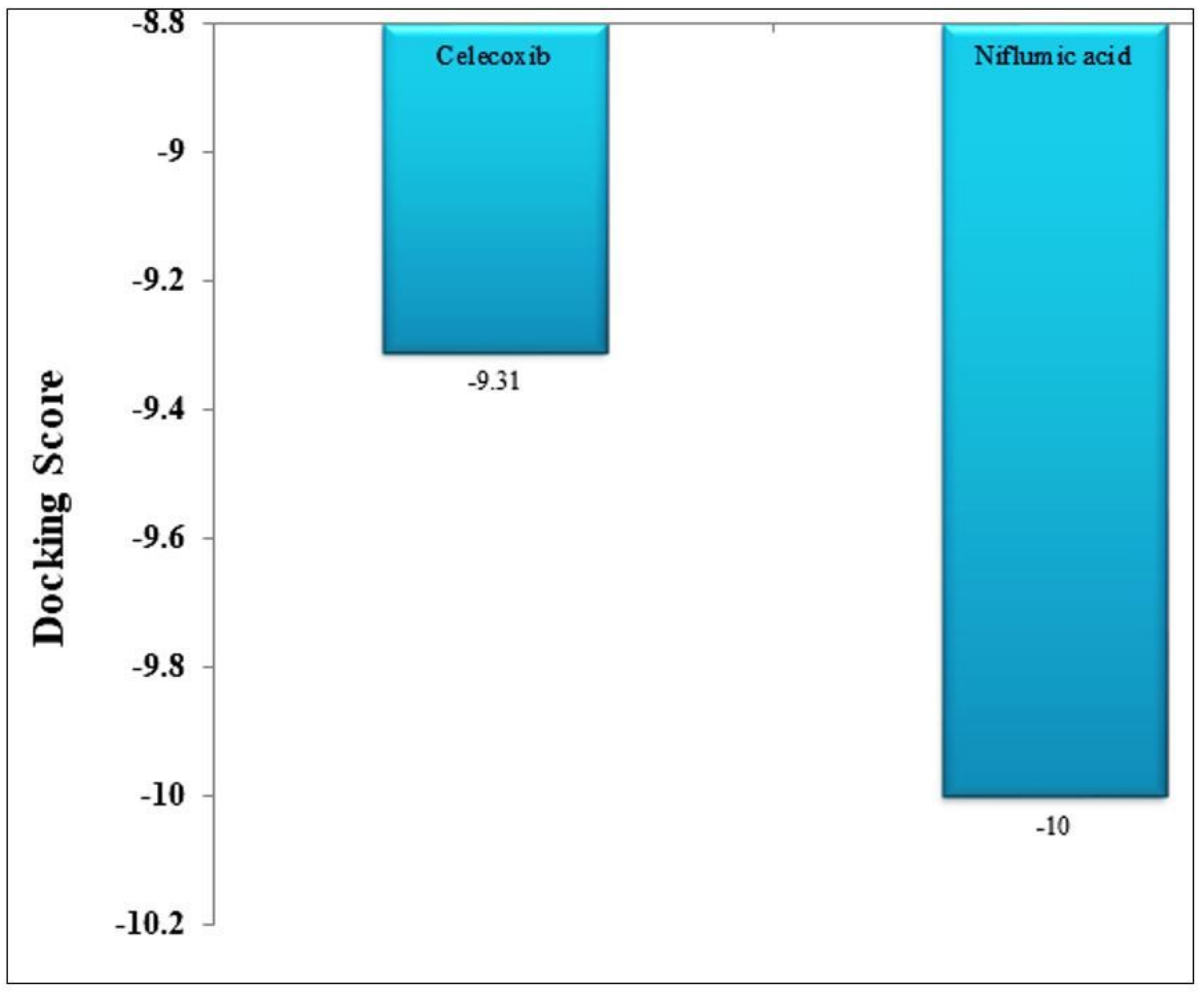

Figure 2

Docking score of celecoxib and niflumic acid against cyclooxygenase-2 (COX-2) enzyme. When epik state penalties are incorporated in scoring it gives rise to docking score. If epik penalties are not included in scoring then docking score and Glide score are identical. However, the default parameters of Glide docking tool include epik state penalty thus it is wise to consider docking score over Glide score. Thus docking score integrates Glide score with epik state penalties. Further, the lowest docking score (more negative) value represents more effective binders as this score simulates binding free energy. 


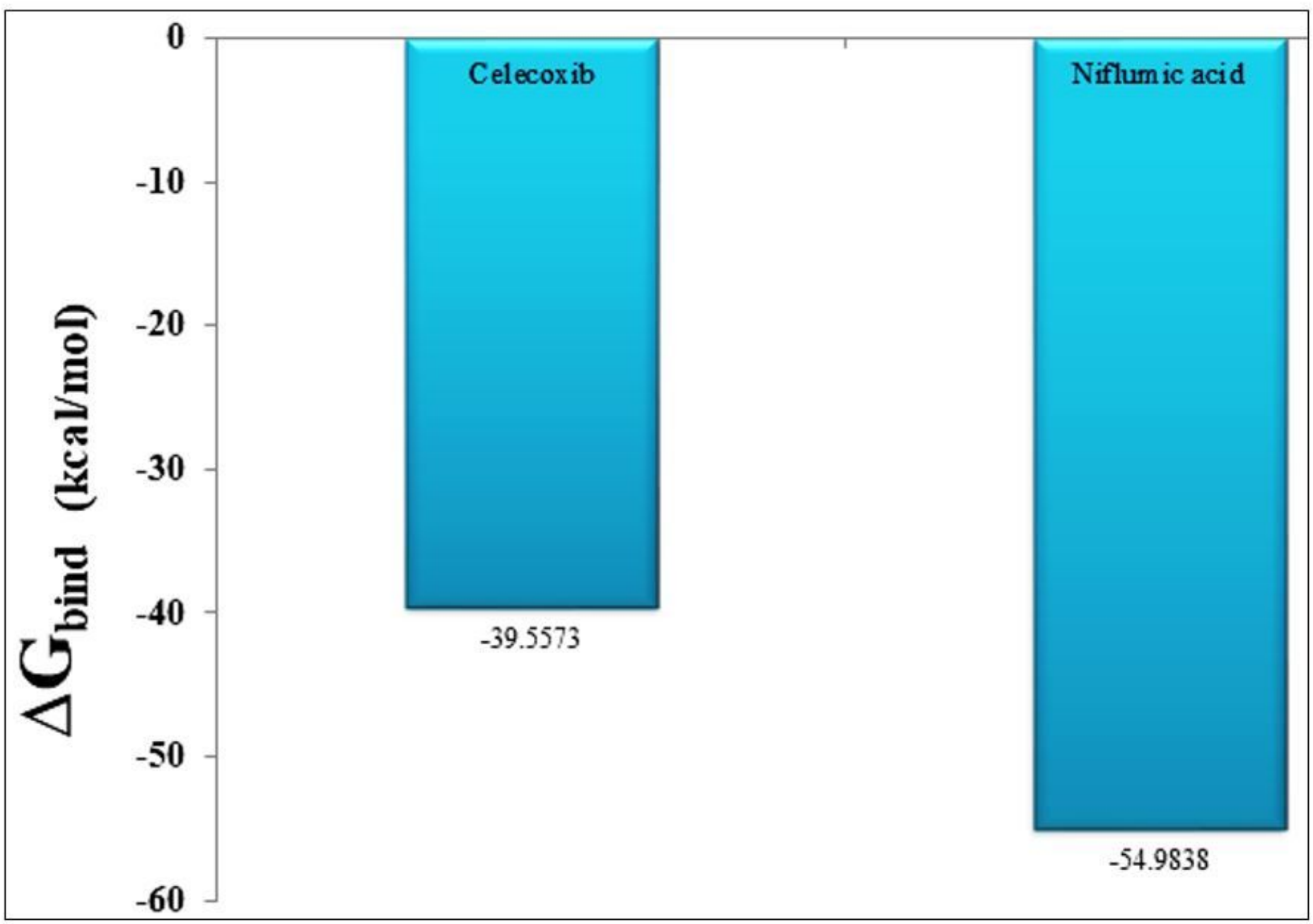

Figure 3

Binding free energy values followed same trend as was observed during molecular docking. Further the estimations of binding free energy performed through MM-GBSA are quite reliant over docking score. Lowest value of binding free energy means stronger binding inclinations while lesser negative binding free energy showing molecules are frail binders. 

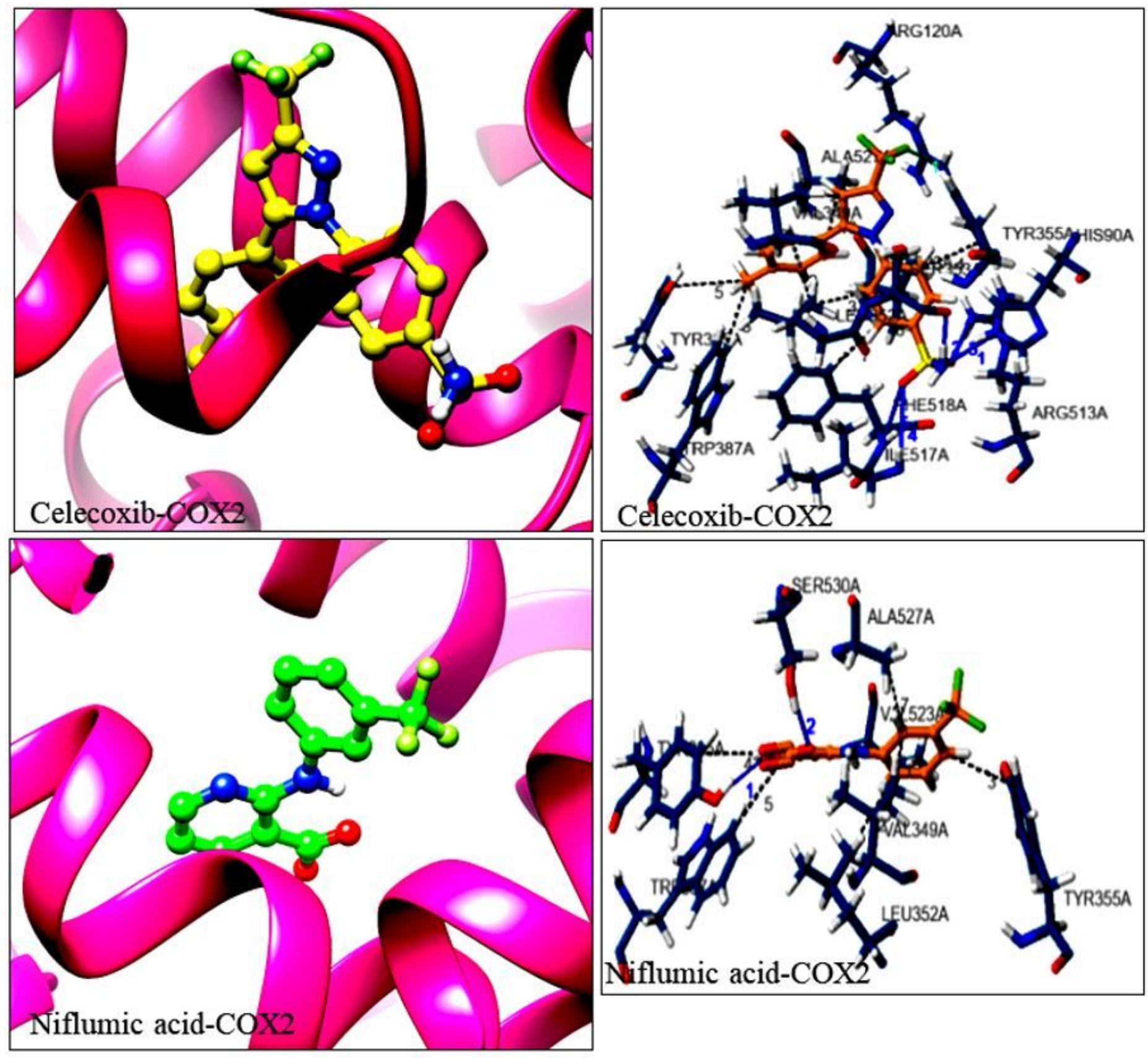

Figure 4

Celecoxib and niflumic acid in the binding groove of COX-2 and their interactions with its active site amino acid residues. Mainly hydrophobic and hydrogen bonding interactions were observed between celecoxib/niflumic acid and COX-2. However, more contacts were seen between celecoxib and COX-2. One halogen bond between was also observed in case of celecoxib-COX2. 

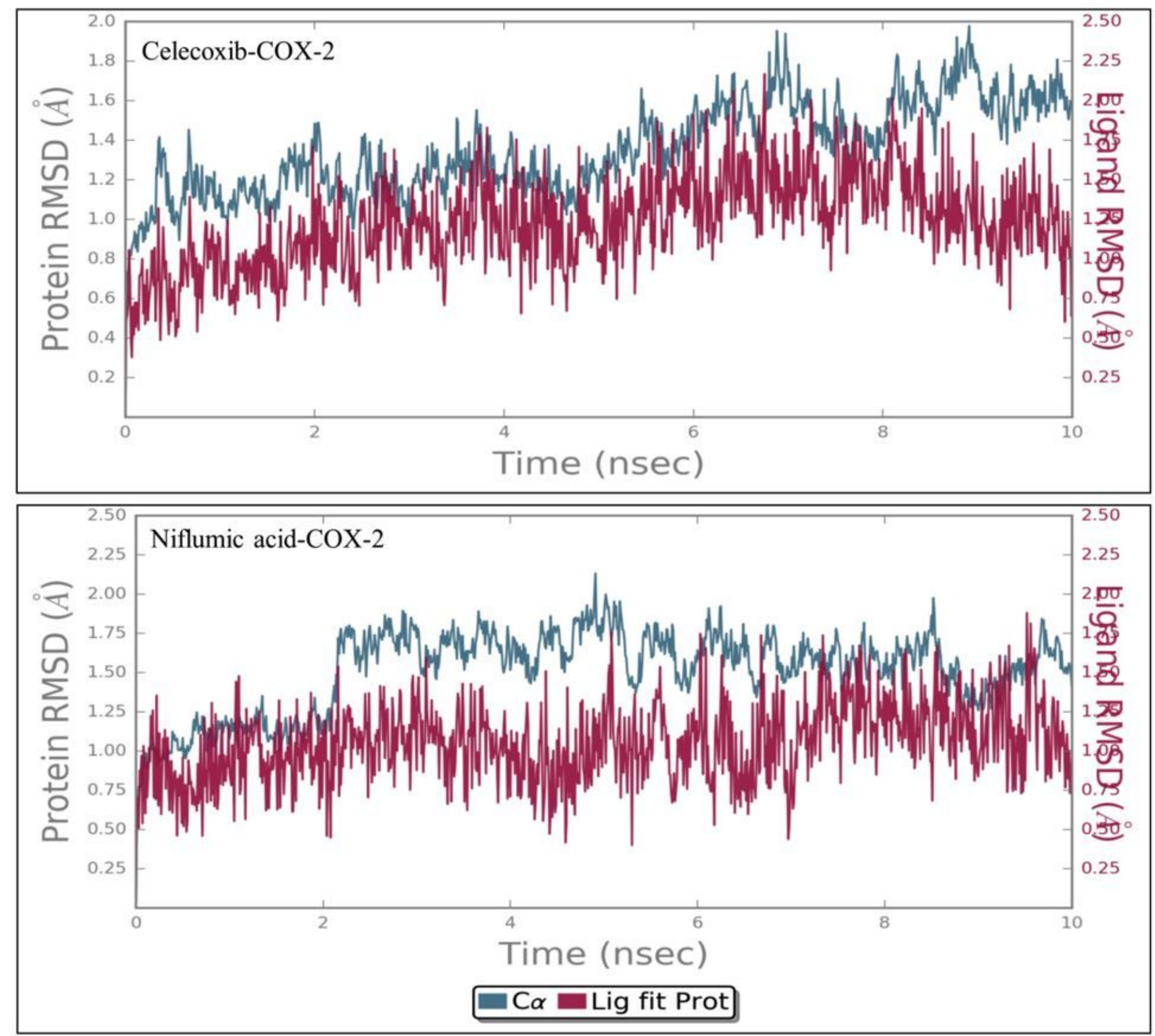

\section{Figure 5}

Protein and ligand RMSD values throughout the simulation time. The top figure indicates COX-2 RMSD on left Y-axis and ligand (celecoxib) RMSD on right Y-axis. Monitoring COX-2 RMSD sheds light on structural conformation the enzyme undergoes all through the simulation duration. RMSD values ranging from 1-3 $\AA$ are strongly acceptable for globular proteins small in size. Values above the provided range suggest that the protein is undergoing an immense conformational change. Ligand RMSD provides insights about the stability of ligand in the binding pocket of protein. The RMSD values substantially higher than protein RMSD indicates the drifting of ligand from the original binding site. From the above figures it is quite evident that both celecoxib and niflumic acid are not diffusing away from the binding pocket of COX-2. However, it is quite evident that niflumic acid is relatively more stable with respect to 
COX-2 than celecoxib. This crux has been derived as the niflumic acid manifested lesser fluctuations than celecoxib.
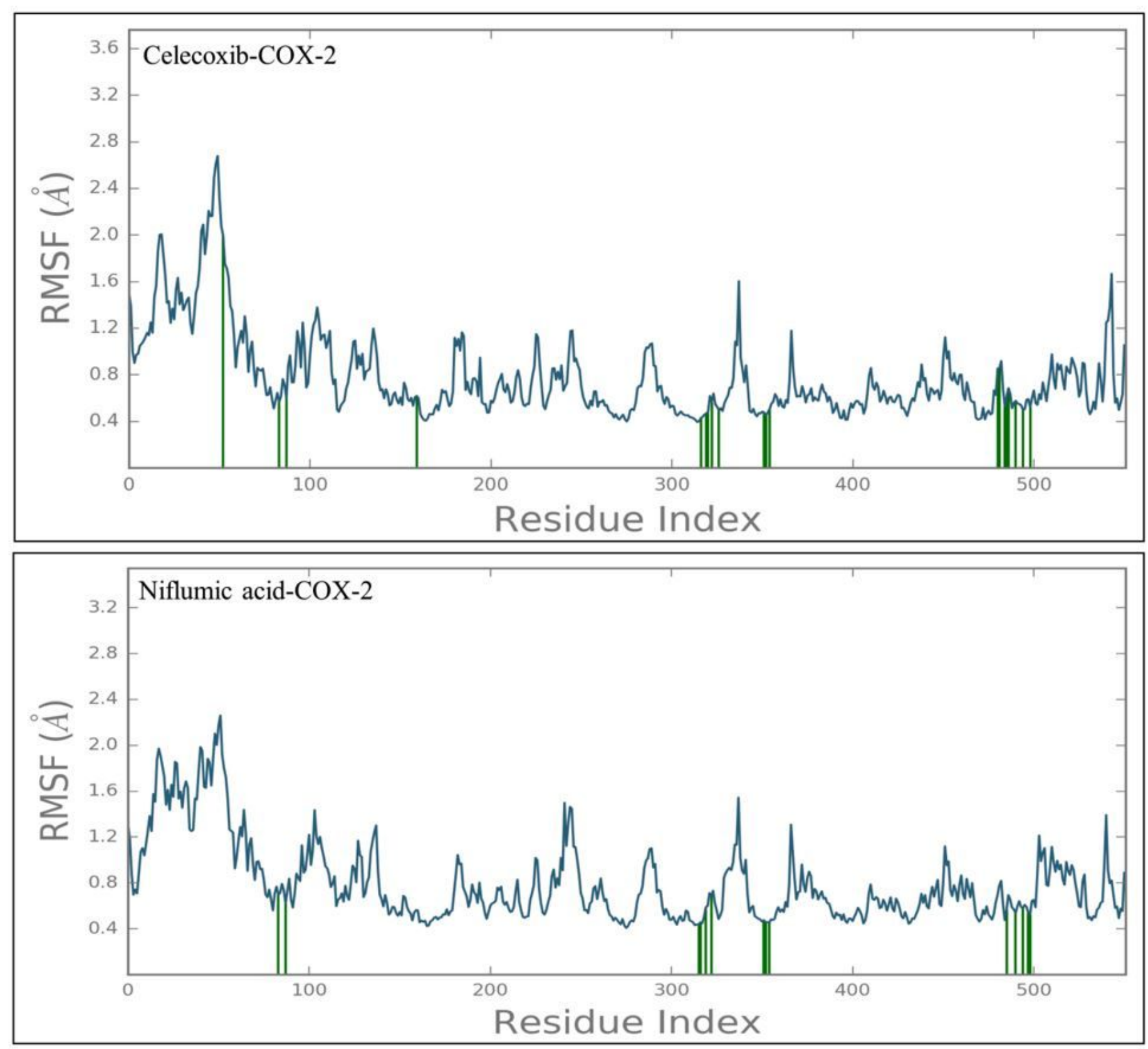

Figure 6

For characterizing local changes root mean square fluctuation (RMSF) has importance. Peaks designate regions that fluctuate highly during the simulation. It is typical that amino and carboxyl terminal regions fluctuate more than the rest of the protein. Beta strands and alpha helices are often rigid than the loop region and thus fluctuate relatively lesser than this unstructured region. Green vertical bars represent the 
amino acid residues of proteins interacting with ligands (celecoxib and niflumic acid). From the above figures it is clear that COX-2 fluctuates relatively more in docked state with celecoxib than niflumic acid.
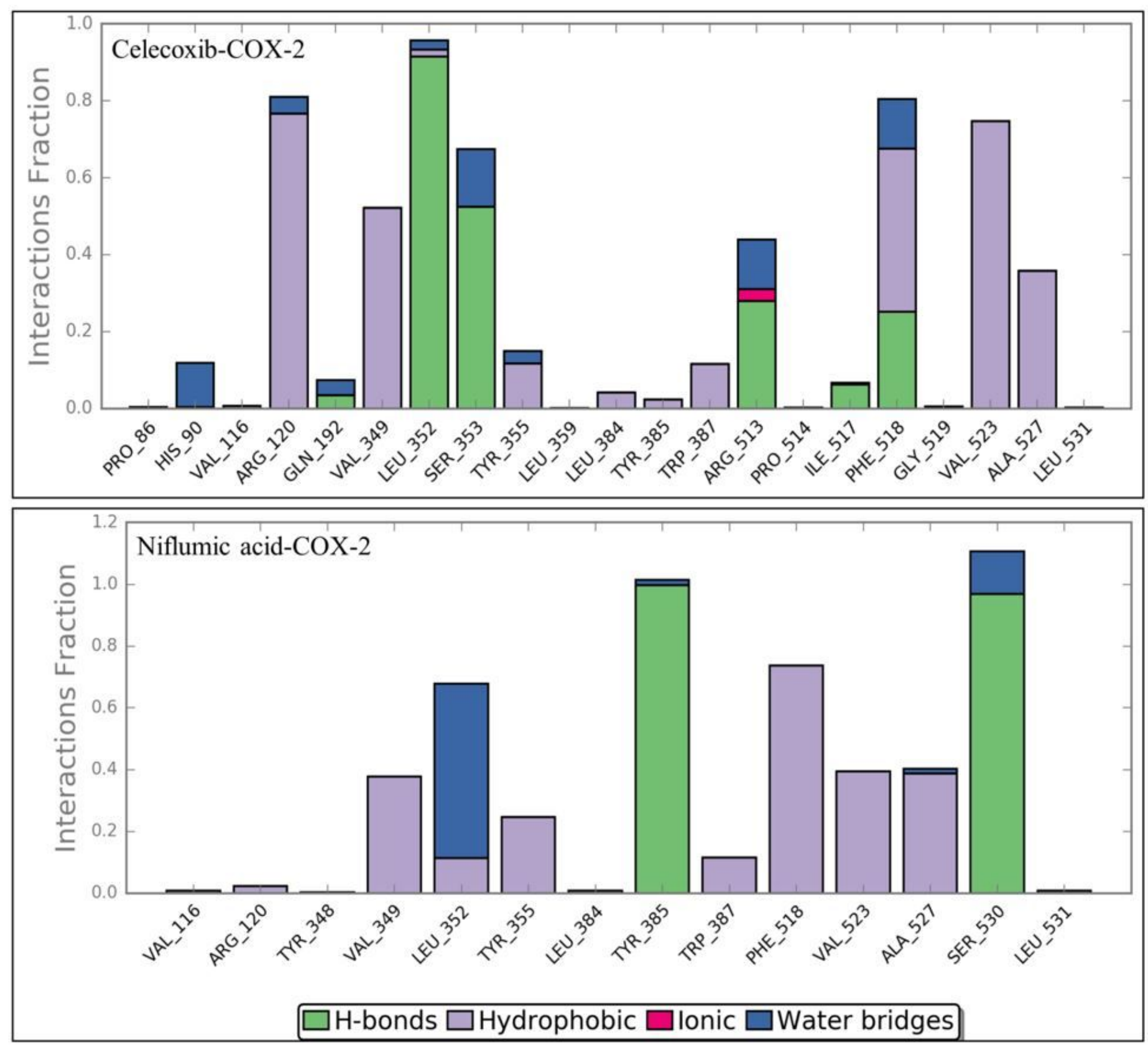

Figure 7

Protein-ligand interactions during the entire simulation time. These interactions may be hydrophobic, hydrogen bond, ionic bonds and water bridges. The stacked bar charts indicate the time point for which a particular interaction is maintained. A given residue may form multiple interactions with the ligand. A value of 1.0 indicates that particular interaction sustains for $100 \%$ of the simulation duration. Even value above 1 are possible due to many interactions between a given residue and ligand. While in case of 
celecoxib-COX-2 LEU 352, ARG 120, SER 353, PHE 518 and VAL 523 are crucial for interaction, the residues SER 530, TYR 385, PHE 518 and LEU 352 are critical for interaction with niflumic acid.

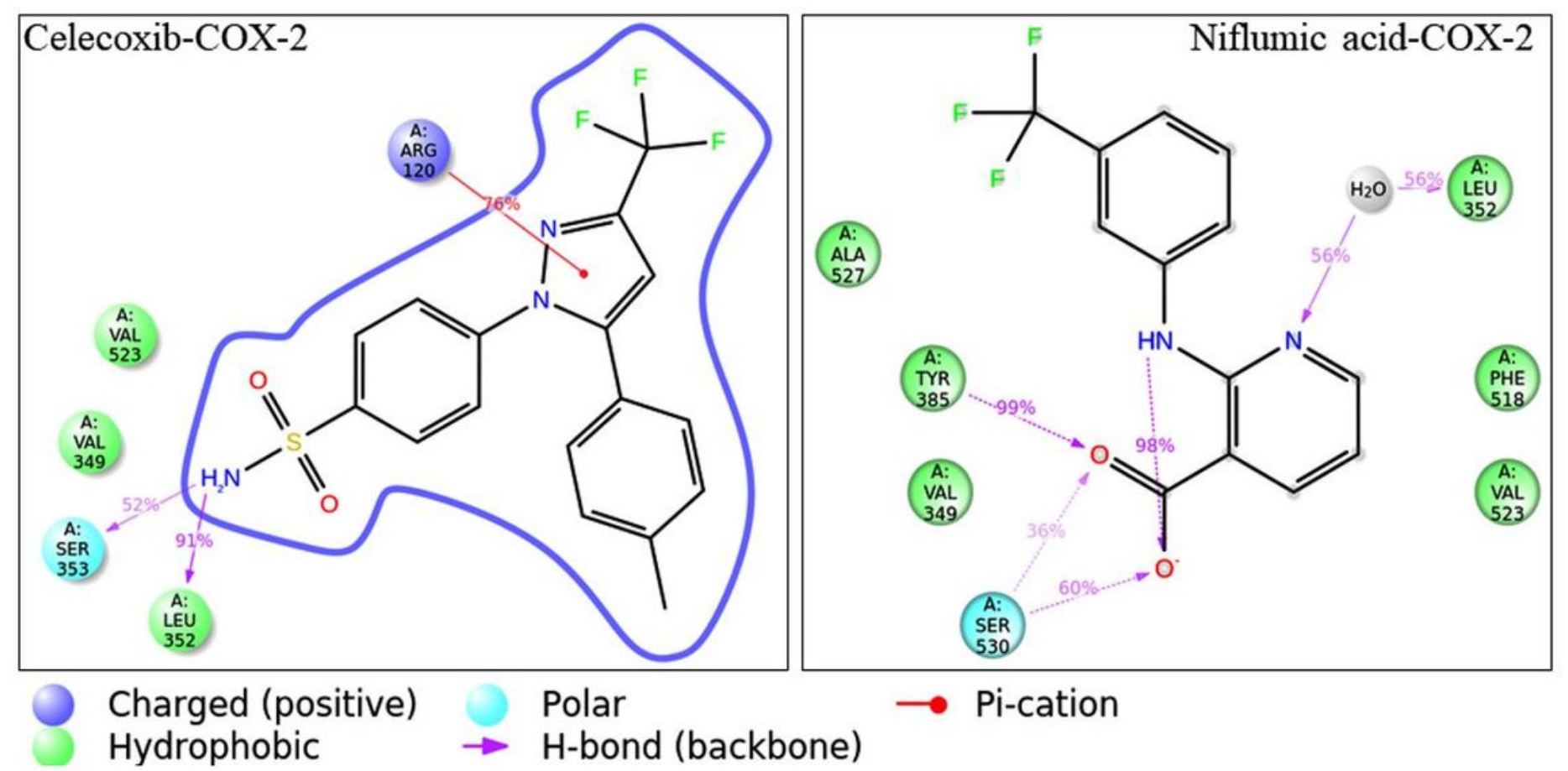

Figure 8

Ligand-protein interactions withstanding beyond $30 \%$ of the simulation time. It is quite evident that both ligands are stable in the active site of COX-2. This is evident as certain interactions both in case of celecoxib-COX-2 and niflumic acid-COX2 remain intact over $50 \%$ of the simulation time. However, niflumic acid demonstrated better stability than celecoxib as one interaction (TYR 385) sustained for $99 \%$ of the simulation time while in case of celecoxib the maximum sustaining interaction with LEU 352 lasted for $91 \%$ of the simulation time only. Thus niflumic acid has better stability over celecoxib in the binding site of COX-2. 